\title{
100 ans de géographie du commerce
}

\author{
(100 YEARS OF RETAIL GEOGRAPHY)
}

\section{Bernadette MÉRENNE-SCHOUMAKER*}

RÉSUMÉ - Pour traduire l'évolution de la discipline, nous avons cherché à comprendre les facteurs de son essor depuis 50 ans ainsi que les différents courants de recherche qui s'y sont rencontrés. L'article propose aussi un bilan en termes de forces et de faiblesses.

Mots-clés : Géographie du commerce de détail.

ABSTRACT - To reflect the evolution of the discipline, we have sought to understand the factors behind its growth over the past 50 years and the different research streams that have come together. The article also provides an assessment in terms of strengths and weaknesses.

Key words: Retail Geography.

Cinq numéros du BAGF ont été consacrés (en tout ou en partie) au commerce de détail. Les thèmes abordés sont divers : commerce et aménagement (1993-3), mondialisation de la distribution (2000-1), commerce et centralités dans les très grandes villes mondiales (2001-4), commerce et développement durable (2007-4) et commerce(s) et loisirs (2018-1) ; quatre numéros ont été initiés par $\mathrm{A}$. Metton et le cinquième par $\mathrm{C}$. RenardGrandmontagne.

$\mathrm{Si}$ les articles réunis dans ces cinq volumes sont divers quant aux thématiques ou aux échelles d'analyse, ils ne traduisent toutefois pas toute la diversité des travaux de la géographie du commerce en langue française : plus de 1300 références $^{1}$ d'après un inventaire effectué en 2016 en vue de l'établissement d'une bibliographie thématique et publié par la Commission géographie du commerce du CNFG [Deprez \& al. 2017].

C'est la raison pour laquelle, nous avons décidé d'élargir nos propos à l'ensemble des travaux publiés en cherchant à éclairer l'évolution de la géographie du commerce non sous l'angle purement historique mais davantage

\footnotetext{
* Professeur de Géographie économique, fondamentale et appliquée, Bernadette Mérenne-Schoumaker est spécialiste de géographie économique et de didactique de la géographie Elle est aujourd'hui Professeur émérite de l'Université de Liège - Courriel : b.merenne@uliege.be

${ }^{1}$ Cette bibliographie tenue à jour comprend aujourd'hui une cinquantaine de références supplémentaires
} 
sous un angle épistémologique afin de mettre en évidence les modes de fonctionnement de ce sous-domaine de la géographie. Nous nous interrogerons aussi sur les raisons du développement d'une géographie du commerce et nous tenterons un bilan sur ses forces et faiblesses, voire sur ses spécificités et points communs avec les autres sous- domaines de la géographie.

\section{Facteurs pouvant expliquer le développement de la discipline}

Trois facteurs peuvent être évoqués.

\subsection{Un secteur d'activités en profondes mutations}

Les géographes ont toujours été sensibles aux changements d'organisation des territoires, aux mutations des activités, aux transformations des comportements des hommes dans les espaces qu'ils habitent ou qu'ils fréquentent. Il est donc assez logique qu'ils aient porté leur attention sur un des bouleversements majeurs depuis une cinquantaine d'années des trames urbaines et rurales, à savoir les mutations de la fonction commerciale. En effet, depuis les années 1950, le secteur du commerce de détail a été bouleversé à la fois dans ses dimensions offre et demande et ces changements ont eu de profondes répercussions sur les territoires.

Parmi ces changements majeurs, on peut épingler au niveau de l'offre : les changements de concepts et de formats des magasins, les mutations des opérateurs (croissance de grands groupes et internationalisation des opérateurs) ou encore l'essor de l'e-commerce... et au niveau de la demande: les changements des modes de consommation et des mobilités des clients ainsi que la diversification accrue de ces consommateurs ... Quant aux impacts sur les territoires, ils sont bien connus et se manifestent un peu partout, tant dans les grandes villes que les petites villes, tant en milieu urbain qu'en milieu rural et ce un peu partout dans le monde ; il s'agit principalement du développement de la distribution périphérique et en contre partie le déclin de nombreuses polarités traditionnelles.

De tels bouleversements ont multiplié les possibilités d'analyse scientifique et aussi la demande d'opérateurs (publics et privés) qui souhaitent mieux comprendre les changements pour pouvoir intervenir en connaissance de cause.

\subsection{Un champ d'investigation aux potentialités multiples}

Comme nous le montrerons ci-après (2.1.), les recherches développées en géographie du commerce sont multiples et dépendent d'abord des objectifs des travaux : s'agit-il par exemple de chercher à expliquer les organisations spatiales commerciales ou au contraire le rôle des acteurs dans les formes spatiales, comme les clients, les commerçants ou encore les aménageurs ? S'agit-il de tenter de dresser un tableau des appareils commerciaux à un 
moment bien déterminé ou de mettre en évidence une ou des évolutions? S'agit-il de s'interroger sur le commerce sédentaire ou de tenir également compte du commerce ambulant (foires, marchés, tournées) ? Limite-t-on les investigations au commerce formel ou cherche-t-on aussi à intégrer le commerce informel si important dans les pays du Sud ? Enfin, dans le monde d'aujourd'hui, comment cerner l'impact du commerce en ligne ?

Les potentialités de travaux sont donc multiples et ce d'autant plus que l'interdisciplinarité gagne du terrain. Le commerce intéresse en effet d'autres disciplines : urbanisme, sociologie et anthropologie, économie, histoire, sciences politiques ... avec qui les géographes collaborent de plus en plus. En outre, comme dit plus haut, la finalité des travaux n'est pas que scientifique. Beaucoup de recherches ont été commandées et le sont encore actuellement par des entreprises ou des opérateurs immobiliers ou des responsables publics nationaux, régionaux ou locaux comme par exemple en France, les chambres de commerce.

Cette forte demande sociale n'est sans doute pas étrangère à l'essor de la géographie du commerce car elle a apporté à des chercheurs universitaires ce qui leur manque souvent, à savoir des moyens financiers et elle a aussi permis à de nombreux géographes de trouver un emploi dans des organismes publics et le secteur privé ; elle a encore permis à beaucoup de géographes de mieux s'intégrer dans la vie économique et sociale via par exemple leur participation à différentes commissions ou groupes de travail tant en France (Commission Départementale d'Equipement Commercial - CDEC -, puis actuellement Commission Départementale d'Aménagement Commercial - CDAC -) qu'en Belgique (Observatoire du Commerce de la Région Wallonne).

\subsection{Le développement et le dynamisme de la Commission "géographie du commerce »du CNFG}

L'essor de la géographie du commerce en France est encore dû à la création en 1973, à l'initiative de Jacqueline Beaujeu Garnier, alors Professeure à la Sorbonne et conseillère en aménagement pour la région parisienne, de la Commission géographie du commerce du CNFG. Son but était clair : fédérer les géographes qui travaillent sur le commerce, tant au niveau national qu'international et promouvoir la recherche en géographie du commerce.

Depuis près de 50 ans, cette Commission a toujours fonctionné et a tenté de se renouveler. A cette fin, elle a développé trois axes d'action :

- des séminaires d'échanges et de réflexion en particulier sur les outils et méthodes de la recherche en géographie du commerce, séminaires organisés généralement deux fois par an ; depuis une dizaine d'années, on y a adjoint des journées «Jeunes chercheurs» où des doctorants ou des jeunes docteurs viennent présenter et discuter leurs travaux, que ces chercheurs soient géographes ou issus de disciplines proches ; 
- des colloques nationaux et internationaux sur des thèmes variés et dans des lieux différents de France ou d'autres pays ; le côté international de la Commission fut en outre renforcé par la fondation en 1984 de la «Commission internationale de Géographie des Activités commerciales » de l'Union Géographique Internationale (UGI), présidée par Alain Metton et qui a fonctionné jusqu'en 2000 (A. Metton fut aussi président de l'AGF de 1994 à 2001) ;

- de nombreuses publications collectives : 32 à ce jour [voir MérenneSchoumaker \& Soumagne 2019] dont un Dictionnaire du commerce et de l'aménagement [Desse \& al. 2008], une synthèse de 50 ans de travaux [Lestrade \& al. 2016], une bibliographie thématique déjà citée ci-dessus et en cours aujourd'hui une anthologie de la cartographie du commerce et de la consommation.

Sans aucun doute, ce qui fait l'originalité de cette Commission est la volonté de ses membres de collaborer, d'échanger, d'impliquer de nouveaux membres (en particulier les jeunes diplômés), de se partager les rôles de direction et d'animation (la Commission a depuis près de 20 ans une codirection hommefemme) et au final de faire passer les intérêts collectifs avant certains intérêts particuliers.

\section{Approche épistémologique de la discipline}

\subsection{Un développement en quatre étapes}

Si le fait marchand est peu présent dans la géographie française jusqu'à la Seconde Guerre mondiale sauf comme générateur de flux [Vidal de la Blache 1911 ou Brunhes 1925] ou encore d'urbanisation [Allix 1923], la prise en compte du commerce dans les travaux géographiques s'est faite en quatre étapes [Lemarchand \& al. 2014] :

- apparition dans les années 50 sous la forme d'une géographie morphologique (travaux initiés par J. Tricart sur l'analyse de l'habitat et du tissu urbain) et d'une géographie régionale (zones d'influence des villes et réseaux de villes ) (où l'on note l'influence de l'économiste A. Piatier et de géographes belges et français) avec une forte influence de géographes anglosaxons comme R.E. Dickinson ou B. Berry ;

- essor dans les années 60 d'une géographie du commerce avec l'émergence de la géographie appliquée et de l'urbanisme commercial sous l'impulsion de J. Beaujeu Garnier (puis d'A. Metton et de M. Coquery) en France et de J.A. Sporck en Belgique avec de nouveau des liens importants avec la géographie anglaise (notamment R. Davies et les chercheurs de l'Oxford Institute of Retail Management) ;

- élargissement et multiplication des travaux dans les années 1980 et 1990 avec développement d'un nouvel intérêt : le comportement des acteurs ;

BAGF - GÉOGRAPHIES - 2020-1/2 
- affirmation de la discipline et ouverture aux autres disciplines étudiant le commerce depuis les années 2000 avec émergence de nouveaux paradigmes : mondialisation, consommation, développement durable, culture...

À notre sens, les géographes qui ont porté les premiers leur attention au secteur du commerce de détail émanaient principalement de quatre sousdomaines de la géographie : la géographie urbaine, la géographie économique, la géographie appliquée et un peu plus tardivement la géographie sociale et culturelle. La géographie du commerce est ainsi née au carrefour de ces quatre domaines qui l'éclairent sous des angles un peu différents. Les géographes urbains se sont surtout intéressés, à l'échelle méso-et micro-spatiale, à la place du commerce dans les trames urbaines et, à l'échelle macro-spatiale, au rôle du commerce dans la hiérarchie urbaine et les réseaux urbains; les géographes économistes se sont focalisés sur les localisations (facteurs et théories) tandis que les géographes appliqués ont développé l'urbanisme commercial ; quant aux géographes sociaux, ils ont développé les volets comportement de consommation et liens entre commerce et consommation.

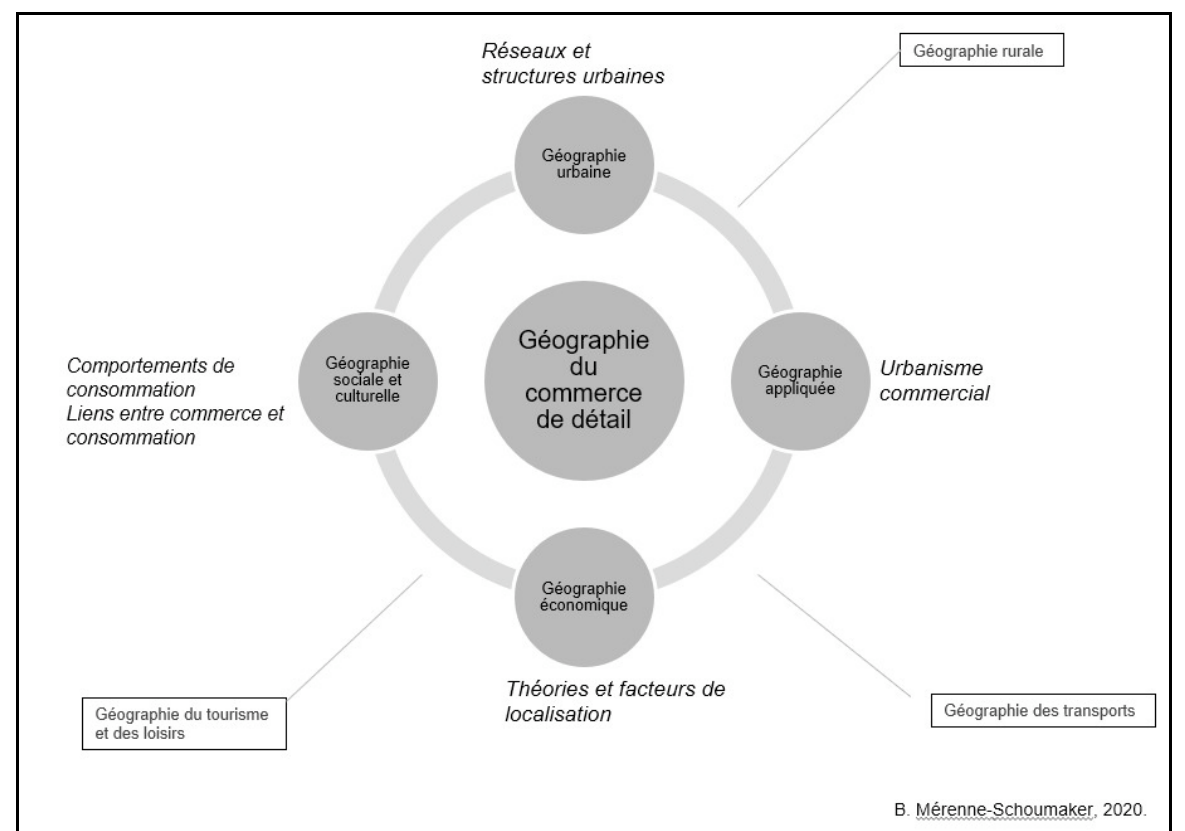

Figure 1 - Liens entre la géographie du commerce de détail et les autres sousdomaines de la géographie

La figure 1 tente de rendre compte des liens entre la géographie du commerce de détail et les autres sous-domaines de la géographie. Nous y avons placé dans un premier cercle les quatre domaines de la géographie qui ont 
contribué à l'émergence de la géographie du commerce (avec leur thématique principale) tandis qu'ont été mis un peu en retrait trois autres domaines ayant des liens plus récents avec la géographie du commerce de détail : la géographie rurale [Pouzenc 2019], la géographie des transports et la géographie du tourisme et des loisirs.

\subsection{Quatre grandes thématiques en termes de recherches académiques}

\subsubsection{Hiérarchie des centres commerçants et aires d'attraction commerciale}

Ce courant de recherche a été développé dès les années 1950-1960. Focalisé à une échelle macro-spatiale, il regroupe des travaux théoriques et empiriques. Cherchant à classer des centres, il repose sur des critères fonctionnels dont le poids et la diversité des commerces. Il a aussi pour objectif de tracer les zones d'influence des centres. A cette fin, il a développé des modèles ou repose sur l'analyse de courbes isochrones et de résultats d'enquêtes. Si de tels travaux conservent toujours de l'intérêt aujourd'hui, il faut admettre que les classements s'avèrent plus difficiles car les situations se sont complexifiées en lien avec les évolutions récentes des appareils commerciaux et les comportements des consommateurs moins contraints par la proximité.

\subsubsection{Tissus commerçants : trames et structures et localisation des commerces}

Il s'agit aussi d'un courant de recherche développé dès les années 19501960 mais contrairement au précédent, il se limite aux échelles micro- et mésospatiales et regroupe des études le plus souvent empiriques des appareils commerciaux. Parmi ses aspects les plus originaux, nous noterons les études en lien avec la rente foncière ou le principe de différenciation minimum, expliquant des regroupements de certaines activités.

\subsubsection{Comportement spatial des consommateurs}

Développé depuis les années 1970-1980, ce courant se focalise comme le précédent sur les échelles micro- et méso-spatiales et regroupe aussi beaucoup de travaux empiriques. Son objectif est d'analyser les comportements des clientèles en prenant en compte le rôle de l'information et des caractéristiques des individus (dont leur mobilité). Il s'attache aussi aux liens entre commerce et consommation, entre commerce et culture. Une des difficultés majeures rencontrées par les recherches de ce groupe est qu'elle impose des lourdes enquêtes et des protocoles rigoureux afin de collecter des informations fiables.

\subsubsection{Stratégie des acteurs et interventions des pouvoirs publics}

Développé également depuis les années 1980-1990, combinant des études 
d'abord empiriques puis théoriques à différentes échelles, les travaux relevant de ce courant visent, d'une part, à analyser les stratégies de localisation des grands groupes nationaux et internationaux avec prise en compte des réseaux des concurrents, des étapes dans la vie d'un réseau et des facteurs caractérisant les opérateurs comme leur origine spatiale ou les produits et services offerts et, d'autre part, les impacts des politiques publiques sur l'organisation et l'évolution des trames commerciales. Certains chercheurs ont aussi tenté de cerner le rôle des opérateurs de l'immobilier commercial.

\subsection{Trois grandes thématiques en termes de recherches appliquées}

\subsection{1.Études d'implantation ou de faisabilité de projets}

C'est un axe déjà ancien (depuis les années 1960-1970) qui répond à une demande émanant au départ de firmes commerciales privées et plus récemment de promoteurs immobiliers. Le géographe est sollicité pour optimaliser le choix de la localisation d'un projet. A cette fin, il s'appuie principalement sur les modèles et facteurs de localisation.

\subsubsection{Geomarketing}

Le courant est plus récent (années 1980-1990). Le commanditeur est aussi une firme commerciale privée qui souhaite disposer d'informations produites par le géographe (bases de données et SIG) afin de l'aider à prendre des décisions. Les problèmes traités se rapportent tout autant au champ du Business to Business (B to B) qu'au champ du Business to Consumer (B to C). Les performances du système sont largement liées à la fiabilité des sources (qualité, mise à jour) et à l'échelle spatiale de saisie des données.

\subsubsection{Urbanisme commercial}

Comme nous l'avons déjà dit, l'idée d'aménager les lieux de commerce et mieux planifier les villes pour et avec le commerce est une préoccupation qui a émergé dès les années 1950-1960 et où, dès le départ, on a fait appel à des géographes. Dans ce cas, la demande émane principalement du secteur public. Les travaux commandés sont de natures diverses à la fois en termes d'objectifs (réaménager un espace existant, planifier un nouvel espace...) et au niveau des échelles spatiales d'analyse (quartier, commune, agglomération, région...). Toutefois, comme beaucoup d'études ne concernent que des espaces restreints, cela ne permet pas toujours de mener les bonnes actions car en matière de commerce de détail, il faut une vue globale de l'espace pouvant être impacté par une opération; par ailleurs, il faut souligner toute la fragilité de la fonction commerciale qui est une fonction induite notamment par les populations fréquentant l'espace concerné et parallèlement les modes de circulation dans cet espace. 


\subsection{Diversité des méthodes}

Les travaux se distinguent encore par les méthodes utilisées à la fois pour la collecte des données et l'analyse et le traitement des informations.

La collecte des données est toujours une étape difficile car l'activité commerciale est généralement peu recensée par les instituts de statistiques ou les organismes professionnels et de plus le commerce évolue sans cesse. En outre, la définition même de l'activité varie selon les auteurs, d'où la nécessité d'arrêter une classification si l'on veut pouvoir comparer des espaces différents ou le même espace à des moments différents de son histoire. Le problème de la classification des points de vente et des méthodes de prise des informations (relevés sur le terrain de magasins, enquêtes auprès des clients, des commerçants, des promoteurs, des responsables publics)... a été une préoccupation majeure de la Commission de commerce de détail du CNFG.

Pour analyser et traiter les données, les géographes ont adopté au départ des méthodes proches de celles de la démographie. Puis certains ont eu recours aux méthodes de l'analyse spatiale. La cartographie a toujours été au cœur des travaux qui profitent de plus en plus des progrès liés à l'informatisation des données et aux apports des SIG.

\section{Essai de bilan}

\subsection{Forces}

Nous en voyons cinq :

- une géographie ancrée dans la structure spatiale des territoires et dans le comportement des populations ;

- une géographie de plus en plus ouverte à des enjeux sociétaux : mondialisation, mobilité et impacts environnementaux, dualisation des sociétés ;

- une géographie de plus en plus intéressée par l'ailleurs et qui réunit des chercheurs tant du Nord que du Sud;

- une géographie qui tisse de plus en plus de liens avec les autres disciplines ;

- une géographie qui conforte l'utilité sociale des géographes.

\subsection{Faiblesses}

Mais cette géographie présente aussi trois faiblesses :

- une géographie souvent plus empirique que théorique

- peu de traités généraux en langue française alors que l'on en compte plus de dix en langue anglaise ;

- des études qui ont trop souvent isolé la fonction commerciale de ses environnements. 
En fait ces forces et ces faiblesses se retrouvent dans beaucoup d'autres sous-domaines de la géographie, notamment de la géographie humaine.

\section{Conclusion}

Nous conclurons avec deux citations :

- «Le commerce est étroitement lié à toute forme de société humaine. Il en reflète les caractéristiques de même qu'il contribue à en façonner l'évolution (...) Le géographe est bien placé pour étudier les activités commerciales aussi bien dans leur implantation que dans leur fonctionnement. Son souci des "milieux» complexes formés par l'imbrication de l'espace et de la société lui permet de traiter à la fois des apparences et des structures » [Beaujeu-Garnier in Metton (dir.) 1984, p.12].

- «Le commerce par sa très grande réactivité aux changements apparait comme un révélateur pertinent des mutations et des paradoxes qui affectent la dynamique des territoires. Il n'en est pas pour autant le moteur »[Bondue 2000, p. 100].

Ainsi, le développement des études de géographie commerciale a permis sans nul doute de mieux comprendre non seulement le fonctionnement économico-spatial mais aussi socio-spatial de notre monde.

\section{Éléments de bibliographie}

- ALLIX, A. (1923) - «Les foires, étude géographique », La Géographie, t. 39, n 5, mai, $43 \mathrm{p}$.

- BEAUJEU-GARNIER, J. \& DELOBEZ, A. (1977) - La géographie du commerce, Paris, Masson, $262 \mathrm{p}$.

- BONDUE, J.-P. (2000) - «Le commerce dans la géographie humaine », Annales de Géographie, $\mathrm{n}^{\circ}$ 611, pp. 94-102. https://www.persee.fr/doc/geo_00034010_2000_num_109_611_1907

- BRUNHES, J. (1925) - La géographie humaine, Paris, Librairie Félix Alcan, 3 volumes, XI + 574 p. ; 575 à 974 p. ; atlas: 3 feuillets, 278 fig. 162 p.

- COQUERY, M. (1977) - Mutations et structures du commerce de détail en France, Cergy, Le Signe, $1440 \mathrm{p}$.

- DAVIES, R.L. (1976) - Marketing Geography with Special Reference to Retailing, Corbridge, Retailing and Planning Association, 300 p.

- DEPREZ, S., LESTRADE, S., MÉRENNE-SCHOUMAKER, B., DESSE, R-P. \& SOUMAGNE, J. (coédit. scientifiques) (2017) - Commerce, Géographie et Aménagement. Bibliographie thématique en géographie du commerce - Les terrains de la géographie du commerce depuis un demi-siècle, CNFG - Commission géographie du commerce, $217 \mathrm{p}$. http://www.cnfg.fr/images/stories/docs_com_commerce/2017/COMMERCE-

GEOGRAPHIE-ET-AMENAGEMENT.pdf

- DESSE, R-P., FOURNIÉ, A., GASNIER, A., LEMARCHAND, N., METTON, A. \& SOUMAGNE, J., (dir.) (2008) - Dictionnaire du commerce et de l'aménagement, Rennes, Presses Universitaires de Rennes, $357 \mathrm{p}$.

- DICKINSON, R.E. (1929-30) - «The Regional Functions and Zones of Influence of Leeds And Bradford », Geography, vol. 15, n 7 pp. 548-557. 
- DICKINSON, R.E. (1934) - « Markets and Market Areas of East Anglia », Economic Geography », vol. 10, pp. 172-182.

- LEMARCHAND, N., MÉRENNE-SCHOUMAKER, B. \& SOUMAGNE, J. (2014) -

"La difficile émergence de la géographie du commerce ", in Le commerce dans tous ses états. Espaces marchands et enjeux de société, Rennes, Presses Universitaires de Rennes, Coll. Espace et Territoires, pp. 13-20, http://hdl.handle.net/2268/168423

- LESTRADE, S., MÉRENNE-SCHOUMAKER, B., DESSE, R.-P., SOUMAGNE, J. \& DEPREZ, S. (coord. scientifiques) (2016) - «Un demi-siècle de recherches en géographie $\mathrm{du}$ commerce », Bulletin de la Société géographique de Liège, $\mathrm{n}^{\circ}$ 66, https://popups.uliege.be/0770-7576/index.php?id=4156

- MÉRENNE-SCHOUMAKER, B. (1987) - Géographie et analyse du commerce urbain : bilan et perspectives. Recherches de Géographie Urbaine, Hommage au Professeur J.A. Sporck, Liège, Société géographique de Liège et Presses Universitaires de Liège, pp. 325335. http://hdl.handle.net/2268/72162

- MÉRENNE-SCHOUMAKER, B. \& DEPREZ, S. (2016) - « La géographie du commerce de détail : outils et méthodes », in Un demi-siècle de recherches en géographie $d u$ commerce, BSGLg, Bulletin de la Société géographique de Liège, $\mathrm{n}^{\circ}$ 66, pp. 13-17. http://popups.ulg.ac.be/0770-7576/index.php?id=4156 et http://hdl.handle.net/2268/21245

- MÉRENNE-SCHOUMAKER, B. \& SOUMAGNE, J. (2019) - Liste des colloques et publications collectives de la Commission géographie du commerce, CNFG, 2019 http://www.cnfg.fr/liste-des-colloques-et-publications-collectives/

- METTON, A. (1980) - Le commerce et la ville en région parisienne, petits commerces, marchés, grandes surfaces et centres commerciaux, Courbevoie, auteur-édit., 567 p.

- METTON, A. (dir.) (1984) - Le commerce urbain français » Paris, PUF , 280 p.

- PIATIER, A. (1956) - « Les attractions commerciales des villes, une nouvelle méthode de mesure », Revue juridique et économique du Sud-Ouest, $\mathrm{n}^{\circ}$ 4, pp. 575-602.

- POUZENC, M. (2019) - Commerce et ruralité. La "renaissance rurale » d'un siècle à l'autre?, Toulouse, Presses universitaires du Midi, Coll. Ruralités Nord-Sud, 187 p.

- SPORCK, J. A. (1964) - «Etude de la localisation du commerce de détail. Aspects méthodologiques », Bulletin de la Société belge d'Etudes géographiques, t. XXXIII, n²1, pp. 53-106.

- TRICART, J. (1951) - L'habitat urbain, problèmes et méthodes, Paris, Centre de Documentation Universitaire, 295 p. ronéo.

- VIDAL DE LA BLACHE, P. (1911) - Tableau de la Géographie de la France, (Tome 1 de l'Histoire de France depuis les origines jusqu'à la Révolution, dir. E. Lavisse), Paris, Colin, $394 \mathrm{p}$.

- Site de la Commission géographie du commerce du CNFG : http://www.cnfg.fr/commissions/geographieducommerce/

et aussi :

- hypotheses / Commission géographie et commerce 